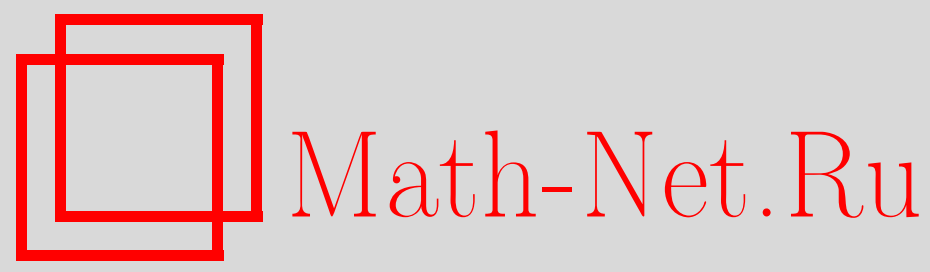

А. В. Лебедев, Экстремумы субэкспоненциального дробового шума, Матем. заметки, 2002, том 71, выпуск 2, 227-231

DOI: https://doi.org/10.4213/mzm341

Использование Общероссийского математического портала Math-Net.Ru подразумевает, что вы прочитали и согласны с пользовательским соглашением http://www.mathnet.ru/rus/agreement

Параметры загрузки:

IP : 54.89 .56 .158

26 апреля 2023 г., 18:12:24

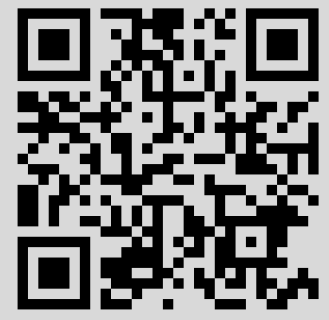




\section{ЭКСТРЕМУМЫ \\ СУБЭКСПОНЕНЦИАЛЬНОГО ДРОБОВОГО ШУМА}

\section{А. В. Лебедев}

Рассмотрено асимптотическое поведение экстремумов дробового шума в случае монотонных функций отклика с неограниченньм носителем и субэкспоненциально распределенных амплитуд. Предполагается, что распределение амплитуд принадлежит области притяжения некоторого максимум-устойчивого закона. Получено предельное распределение для максимумов.

Библиографой: 12 названий.

1. Введение. Исследования дробового шума восходят к началу XX века [1]. Фундаментальной в этой области считается работа [2]. Помимо описания флуктуационных шумов в электрических цепях в дальнейшем были найдены приложения модели в самых разных областях: акустике, оптике, механике, биологии, метеорологии, гидрологии, нейропсихологии, теории риска (страховании) и др.

Под дробовым шумом будем понимать случайньй процесс вида

$$
X(t)=\sum_{t_{n} \leqslant t} \xi_{n} \varphi\left(t-t_{n}\right), \quad t \geqslant 0,
$$

где случайные величины $\xi_{n}, n \geqslant 1$, назьваемые амллитудамu, независимы и одинаково распределены, $t_{n}, n \geqslant 1$, образуют процесс восстановления, причем $\left\{\xi_{n}\right\}$ и $\left\{t_{n}\right\}$ независимы; $\varphi(t)$ - измеримая функция на $\mathbb{R}_{+}$, называемая функиией отклика. Функции распределения амплитуд $\xi_{n}$ и промежутков $\tau_{n}=t_{n}-t_{n-1}, t_{0}=0$, обозначим через $A$ и $G$ соответственно.

Нас будет интересовать асимптотическое поведение процесса $M(t)=\sup \{X(u)$ : $u \in[0, t]\}$ при $t \rightarrow \infty$.

В [3] изучались максимумы процессов с правильно меняющимися хвостами распределений амплитуд и финитными функциями отклика. Случай функций отклика с неограниченньм носителем (в частности, показательной) изучался в [4], но для единичных амплитуд.

Проведем обобщение модели [3], рассмотрев случай монотонных функций отклика с неограниченньм носителем и субэкспоненциально распределенных амплитуд.

Субэкспоненциальные распределения были введены в [5] как удовлетворяющие следуюшим условиям:

1) $F(x)=0, x<0$;

Работа выполнена при финансовой поддержке Российского фонда фундаментальных исследований, грант № 00-01-00131. 
2) $F(x)<1, x>0$;

3) $\bar{F}^{2 *}(x) \sim 2 \bar{F}(x), x \rightarrow \infty$, где $\bar{F}(x)=1-F(x)$.

Последнее условие оказьвается эквивалентно $\bar{F}^{n *}(x) \sim n \bar{F}(x), x \rightarrow \infty, n \geqslant 2$. Современное состояние вопроса изложено в обзоре [6]. Интересные результаты были получены в [7], [8].

В классе субэкспоненциальных распределений $S$ выделим подкласс $S_{\max }$ распределений, принадлежащих области притяжения какого-либо невырожденного максимумустойчивого закона $H$ при линейной нормировке. Иначе говоря, для таких распределений существуют числовые последовательности $a_{n}>0, b_{n}, n \geqslant 1$, такие, что

$$
\lim _{n \rightarrow \infty} \mathrm{P}\left(\max \left\{\xi_{1}, \ldots, \xi_{n}\right\} \leqslant a_{n} x+b_{n}\right)=H(x) .
$$

K данному классу относятся, например, распределения Парето, Вейбулла (с показателем меньше единищы), Барра, Бенктандера (I и II типов), логарифмически нормальное, логарифмически гамма и др. Принадлежность области притяжения означает наличие регулярности, без которой получение многих важных результатов усложняется.

Фундаментальной в теории экстремумов является работа [9] о трех типах максимумустойчивьх законов (распределений экстремальных значений) и их областях притяжения. Более современное состояние теории отражено в монографии [10].

Распределения из $S_{\max }$ либо имеют правильно меняющиеся хвосты, либо принадлежат области притяжения двойного показательного закона $\Lambda(x)=\exp \left\{-e^{-x}\right\}[6]$.

Будем далее пользоваться понятием ассоциированности случайных величин [11], [12] (в теории надежности употребляется термин “связность"). Функцию многих переменных $f(x)$, где $x=\left(x_{1}, \ldots, x_{n}\right)$, назовем монотонно неубывающей, если из $x_{i}^{\prime} \leqslant x_{i}^{\prime \prime}$, $1 \leqslant i \leqslant n$, следует $f\left(x^{\prime}\right) \leqslant f\left(x^{\prime \prime}\right)$. Случайные величины набора $\zeta=\left(\zeta_{1}, \ldots, \zeta_{n}\right)$ называются ассочиированными, если $\operatorname{Cov}(f(\zeta), g(\zeta)) \geqslant 0$ для всех тех монотонно неубывающих $f$ и $g$, для которых эта ковариация существует.

Согласно [11] независимые случайные величины ассоциированы, монотонно не убывающие функции от ассоциированных случайных величин также обладают этим свойством, и для ассоциированных $\zeta_{i}$ и любьх чисел $s_{i}$, где $1 \leqslant i \leqslant n$, верно неравенство [11, теорема 5.1]

$$
\mathrm{P}\left(\zeta_{i} \leqslant s_{i}, 1 \leqslant i \leqslant n\right) \geqslant \prod_{i=1}^{n} \mathrm{P}\left(\zeta_{i} \leqslant s_{i}\right) .
$$

2. Основные результаты. Всюду в дальнейшем будем считать выполненными следуюшие предположения:

1) случайные величины $\xi_{n}$ в (1) имеют функцию распределения $A \in S_{\max }$ и

$$
\lim _{n \rightarrow \infty} \mathrm{P}\left(\max \left\{\xi_{1}, \ldots, \xi_{n}\right\} \leqslant a_{n} x+b_{n}\right)=H(x) ;
$$

пусть $\alpha=+\infty$, если $A$ принадлежит области притяжения $\Lambda$, и $\alpha-$ показатель правильно меняющейся функции $\bar{A}$ в противном случае;

2) $\varphi$ неотрицательна, монотонно не возрастает и $\varphi^{\delta}$ непосредственно интегрируема по Риману для некоторого $\delta \in(0, \min \{1, \alpha\}) ; \varphi(0)=1$;

3) $G$ имеет конечное среднее $\gamma>0$; обозначим $\lambda=1 / \gamma$.

Заметим, что условие $\varphi(0)=1$ вместо $\varphi(0) \in(0,+\infty)$ не является ограничительным (поскольку всегда можно нормировать амплитуды соответствующим образом), однако упрощает вычисления. 
Теорема 1. Последовательность $X\left(t_{n}\right)$ имеет предельное распределение $\Psi$ при $n \rightarrow \infty$, и существует распределение $F_{0} \in S$ такое, ито $\bar{\Psi}(x) \leqslant \bar{F}_{0}(x)$ u $\bar{F}_{0}(x) \sim$ $C \bar{A}(x), x \rightarrow \infty$, для некоторого $C>0$.

ТЕОРема 2. Пусть задано семейство числовых последовательностей $u_{n}=u_{n}(s)$, $s>0, n \geqslant 1$, таких, что $n \bar{A}\left(u_{n}\right) \rightarrow s, n \rightarrow \infty$. Тогда

$$
\lim _{t \rightarrow \infty} \mathrm{P}\left(M(t) \leqslant u_{[\lambda t]}\right)=e^{-s} .
$$

СлЕДСТВИЕ. Имеет место соотношение

$$
\lim _{t \rightarrow \infty} \mathrm{P}\left(M(t) \leqslant a_{[\lambda t]} x+b_{[\lambda t]}\right)=H(x) .
$$

ЗАмЕчАниЕ. Существуют субэкспоненциальные распределения, не принадлежашие области притяжения ни одного максимум-устойчивого закона при линейной нормировке. Простой пример такого распределения был приведен Ч.М. Голди в частной переписке с автором: полагаем $F(x)=0$ при $x \leqslant 1$ и для всех $n \geqslant 0$

$$
\bar{F}(x)= \begin{cases}e^{-n} x^{-1}, & e^{2 n} \leqslant x \leqslant e^{2 n+1}, \\ e^{n+1} x^{-2}, & e^{2 n+1} \leqslant x \leqslant e^{2 n+2} .\end{cases}
$$

Поскольку указанньй подкласс субэкспоненциальных распределений пока мало изучен, вопрос об истинности теорем 1 и 2 для него остается открытым.

3. Доказательства. Воспользуемся следующими свойствами субэкспоненциальных распределений $[6, \S 5]$.

I. Если $F, F_{i} \in S$ и $\bar{F}_{i}(x) \sim c_{i} \bar{F}(x), x \rightarrow \infty, c_{i} \in(0, \infty), i=1,2$, то $F_{1} * F_{2} \in S$ и $\overline{F_{1} * F_{2}}(x) \sim\left(c_{1}+c_{2}\right) \bar{F}(x), x \rightarrow \infty$.

II. Пусть случайная величина $\kappa$ со значениями в $\mathbb{Z}_{+}$такова, что $\mathrm{M}(1+\varepsilon)^{\kappa}<\infty$ для некоторого $\varepsilon>0$, тогда если $F \in S$, то $F_{\mathrm{rS}}=\mathrm{M}^{\kappa *} \in S$ и $\bar{F}_{\mathrm{rS}}(x) \sim(\mathrm{M} \kappa) \bar{F}(x), x \rightarrow \infty$.

III. Пусть случайные величины $\eta_{n}, n \geqslant 1$, независимы, имеют распределение $F \in$ $S_{\max }$ и заданы числа $c_{n} \geqslant 0, n \geqslant 1$; обозначим распределение $\sum_{n=1}^{\infty} c_{n} \eta_{n}$ через $F_{\text {ws. }}$. Тогда

a) если хвост $F$ правильно меняюшийся с показателем $\alpha$ и для некоторого $\delta<$ $\min \{1, \alpha\}$ верно $\sum_{n=1}^{\infty} c_{n}^{\delta}<\infty$, то $F_{\mathrm{ws}} \in S$ и $\bar{F}_{\mathrm{ws}}(x) \sim\left(\sum_{n=1}^{\infty} c_{n}^{\alpha}\right) \bar{F}(x), x \rightarrow \infty ;$

б) если $F$ принадлежит области притяжения двойного показательного законаи для некоторого $\delta \in(0,1)$ верно $\sum_{n=1}^{\infty} c_{n}^{\delta}<\infty$, где $c_{n} \leqslant 1$ для всех $n \geqslant 1$, то $F_{\mathrm{ws}} \in S$ и $\bar{F}_{\text {ws }}(x) \sim N_{1} \bar{F}(x), x \rightarrow \infty$, где $N_{1}$ - число $c_{n}$, равных единище.

Лемма. Существуют $\theta>0 u \rho \in(0,1)$ такие, что распределение случайной величины $\nu=\max \left\{n: t_{n} \leqslant \theta n\right\}$ удовлетворяет условию $\mathrm{P}(\nu>m)=O\left(\rho^{m}\right)$, $m \rightarrow \infty$.

ДокАЗАТЕЛЬСТво ЛЕммы. Выберем $\varepsilon>0$ такое, что $0<G(\varepsilon)<1$, обозначим $p=1-G(\varepsilon)$. Определим $\tau_{n}^{\varepsilon}=\varepsilon I\left(\tau_{n} \geqslant \varepsilon\right)$ и $t_{n}^{\varepsilon}=\tau_{1}^{\varepsilon}+\cdots+\tau_{n}^{\varepsilon}$, так что $t_{n}^{\varepsilon} / \varepsilon$ имеет распределение $\operatorname{Bi}(n, p)$. Пусть $0<s<p, \theta=\varepsilon s$, тогда

$$
\mathrm{P}\left(t_{n} \leqslant \theta n\right) \leqslant \mathrm{P}\left(t_{n}^{\varepsilon} \leqslant \theta n\right) \leqslant \rho^{n}, \quad \rho=\left(\frac{1-p}{1-s}\right)^{1-s}\left(\frac{p}{s}\right)^{s} \in(0,1) .
$$

Имеем

$$
\mathrm{P}(\nu>m) \leqslant \sum_{n=m+1}^{\infty} \mathrm{P}\left(t_{n} \leqslant \theta n\right)=O\left(\rho^{m}\right), \quad m \rightarrow \infty
$$


ДоКАЗАТЕЛЬСТво ТеОРемЫ 1. Имеем

$$
X\left(t_{n}\right)=\sum_{k=1}^{n} \xi_{k} \varphi\left(t_{n}-t_{k}\right) \stackrel{d}{=} \sum_{k=0}^{n-1} \xi_{k} \varphi\left(t_{k}\right) \nearrow \sum_{k=0}^{\infty} \xi_{k} \varphi\left(t_{k}\right)=X_{\infty}, \quad n \rightarrow \infty
$$

где $\xi_{0} \stackrel{d}{=} \xi_{1}$. Обозначим распределение $X_{\infty}$ через $\Psi$.

Определим

$$
Y=\sum_{n=0}^{\infty} \xi_{n} \varphi(\theta n)
$$

и обозначим распределение $Y$ через $F_{Y}$. Тогда по свойству III получаем $\bar{F}_{Y}(x) \sim$ $C_{Y} \bar{A}(x), x \rightarrow \infty$. Здесь $C_{Y}=\sum_{n=0}^{\infty} \varphi(\theta n)^{\alpha}$, если хвост $A$ правильно меняется с показателем $\alpha$ (причем $\alpha>\delta$, поэтому ряд сходится), и $C_{Y}=\max \{n: \varphi(\theta n)=1\}+1$ в противном случае.

Определим $Z=\xi_{1}+\cdots+\xi_{\nu}$ и обозначим распределение $Z$ через $F_{Z}$. С учетом леммы по свойству II получаем $\bar{F}_{Z}(x) \sim C_{Z} \bar{A}(x), x \rightarrow \infty$, где $C_{Z}=\mathrm{M} \nu<\infty$.

Положим $F_{0}=F_{Y} * F_{Z}$, тогда по свойству I верно $\bar{F}_{0}(x) \sim C \bar{A}(x), x \rightarrow \infty$, где $C=C_{Y}+C_{Z}$.

Имеем $\varphi\left(t_{n}\right) \leqslant 1$ при $n \leqslant \nu$ и $\varphi\left(t_{n}\right) \leqslant \varphi(\theta n)$ при $n>\nu$. Поэтому

$$
\mathrm{P}\left(X_{\infty}>x \mid \nu=k\right) \leqslant \overline{F_{Y} * A^{k *}}(x), \quad k \geqslant 0 .
$$

По формуле полной вероятности получаем $\bar{\Psi}(x) \leqslant \bar{F}_{0}(x)$.

ДокаЗАТЕЛЬСтво теОРемЫ 2. Рассмотрим случайные величины $X_{n}=X\left(t_{n}\right)$ и $M(k, l)=\max \left\{X_{k+1}, \ldots, X_{l}\right\}, M_{n}=M(0, n)$. Из оценки $X_{n} \geqslant \xi_{n}$ следует

$$
\varlimsup_{n \rightarrow \infty} \mathrm{P}\left(M_{n} \leqslant u_{n}\right) \leqslant \lim _{n \rightarrow \infty}\left\{A\left(u_{n}\right)\right\}^{n}=e^{-s} .
$$

Заметим, что $X_{i}, 1 \leqslant i \leqslant n$, ассоциированы как монотонно неубывающие функции от набора независимых случайных величин $\xi_{i},-\tau_{i}, 1 \leqslant i \leqslant n$. Максимумы $M(k, l)$, в свою очередь, представляют собой монотонно неубывающие функции от $X_{i}, k+1 \leqslant i \leqslant l$, и поэтому также ассоциированы. Следовательно,

$$
\mathrm{P}\left(M_{n} \leqslant u_{n}\right) \geqslant \prod_{j=1}^{[n / k]} \mathrm{P}\left(M\left(l_{j-1}, l_{j}\right) \leqslant u_{n}\right), \quad l_{j}=j k, \quad k \in \mathbb{N} .
$$

С помощью свойства I и теоремы 1 получаем

$$
\begin{aligned}
\varliminf_{n \rightarrow \infty} \mathrm{P}\left(M_{n} \leqslant u_{n}\right) & \geqslant \underset{n \rightarrow \infty}{\lim _{j \rightarrow 1}} \prod_{j=1}^{[n / k]} \mathrm{P}\left(M\left(l_{j-1}, l_{j}\right) \leqslant u_{n}\right) \\
& \geqslant \varliminf_{n \rightarrow \infty} \prod_{j=1}^{[n / k]} \mathrm{P}\left(X_{l_{j-1}+1}+\sum_{i=l_{j-1}+2}^{l_{j}} \xi_{i} \leqslant u_{n}\right) \\
& \geqslant \lim _{n \rightarrow \infty}\left\{F_{0} * A^{(k-1) *}\left(u_{n}\right)\right\}^{[n / k]}=\exp \left\{-\left(1+\frac{C-1}{k}\right) s\right\} .
\end{aligned}
$$


Переходя в правой части (4) к пределу по $k \rightarrow \infty$ и учитьвая (3), получаем

$$
\lim _{n \rightarrow \infty} \mathrm{P}\left(M_{n} \leqslant u_{n}\right)=e^{-s} \text {. }
$$

Обозначим $N(t)=\sup \left\{n: t_{n} \leqslant t\right\}$. Имеем

$$
M(t)=\sup _{u \in[0, t]} X(u)=\max _{n=\overline{1, N(t)}} X_{n}=M_{N(t)}
$$

Поскольку $N(t) / t \rightarrow \lambda$ почти наверное, то из (5) и определения $u_{n}$ следует

$$
\lim _{t \rightarrow \infty} \mathrm{P}\left(M(t) \leqslant u_{[\lambda t]}\right)=\lim _{n \rightarrow \infty} \mathrm{P}\left(M_{n} \leqslant u_{n}\right)=e^{-s} .
$$

ДокАЗАтЕЛЬСтво слЕдСтвИЯ. Распределение $H$ принадлежит к одному из экстремальных типов [9], [10] и в любом случае непрерывно, так что функция $h$, обратная к $(-\ln H)$, определена на $(0,+\infty)$. Из условия следует $n \bar{A}\left(a_{n} x+b_{n}\right) \rightarrow H(x), n \rightarrow \infty$, поэтому можно взять $u_{n}(s)=a_{n} h(s)+b_{n}$ и применить теорему 2 .

Автор благодарен Ч. М. Голди, К. Клюппельберг и Д. Клайну (Cline) за внимание и помощь в поиске информации, а также выражает признательность рецензенту за полезные замечания и предложения.

\section{СПИСОК ЦИТИРОВАННОЙ ЛИТЕРАТУРЫ}

[1] Campbell N. R. The study of discontinuous phenomena // Proc. Cambridge Phil. Soc. 1909. V. 15. №1. P. 117-136.

[2] Райс С. Теория флуктуационных шумов // Теория передачи электрических сигналов при наличии помех. М.: ИЛ, 1953. С. 88-238.

[3] McCormick W. P. Extremes for shot noise processes with heavy tailed amplitudes // J. Appl. Probab. 1997. V. 34. №3. P. 643-656.

[4] Doney R. A., O’Brien G. L. Loud shot noise // Ann. Appl. Probab. 1991. V. 1. №1. P. 88-103.

[5] Чистяков В. П. Теорема о суммах положительных случайных величин и ее приложения к ветвящимся случайным процессам // Теория вероятн. и ее примен. 1964. Т. 9. №4. С. 710-718.

[6] Goldie C. M., Klüppelberg C. Subexponential distributions // A Practical Guide to Heavy Tails: Statistical Techniques for Analysing Heavy Tailed Distributions / ed. R. Adler, R. Feldman, M. S. Taqqu. Boston: Birkhäuser, 1998. P. 435-459.

[7] Калашников В. В., Цициашвили Г.Ш. Двусторонние оценки случайных сумм с субэкспоненциальными слагаемыми // Пробл. передачи информации. 1999. Т. 35. № 3. С. 67-79.

[8] Якымив А. Л. Явные оценки для асимптотики субэкспоненциальных безгранично делимых функций распределения // Матем. заметки. 2000. Т. 67. № 2. С. 295-301.

[9] Gnedenko B. V. Sur la distribution limite du terme maximum d'une série aléatoire // Ann. Math. 1943. V. 44. №3. P. 423-453.

[10] Лидбеттер М., Ротсен Х., Линдгрен Г. Экстремумы случайных последовательностей и процессов. М.: Мир, 1989.

[11] Esary J., Prochan F., Walkup D. Association of random variables with applications // Ann. Math. Stat. 1967. V. 38. № 5. P. 1466-1474.

[12] Бахтин Ю. Ю. Закон повторного логарифма для решения уравнения Бюргерса со случайными начальными данными // Матем. заметки. 1998. Т. 64. №6. С. 812-823.

Московский государственный университет им. М.В. Ломоносова

Поступило

E-mail: alebedev@mech.math.msu.su

15.06 .2000 